\title{
Treatment of highly pathogenic filovirus infections using advanced antisense technology
}

\author{
Travis Warren ${ }^{1 *}$, Kelly Warfield ${ }^{1,3}$, Jay Wells ${ }^{1}$, Dana Swenson ${ }^{1,3}$, Candace Lovejoy ${ }^{2,4}$, Patrick Iversen ${ }^{2}$, Sina Bavari ${ }^{1}$ \\ From $16^{\text {th }}$ International Symposium on HIV and Emerging Infectious Diseases \\ Marseille, France. 24-26 March 2010
}

\section{Background}

Filoviruses, comprised of the Ebola viruses (EBOV) and Marburg virus (MARV), are highly pathogenic agents with human case fatality rates up to $90 \%$. Periodic filovirus outbreaks occur in endemic regions of subSaharan Africa, and a new pathogenic EBOV species was discovered as recently as 2008 , following an outbreak in Uganda. Additionally, these viruses are considered Category A biodefense pathogens. There exists an urgent need to develop effective antiviral therapeutics and vaccines to protect both civilian and military populations at risk of exposure. Our aim was to identify and develop effective antisense-based therapeutics for treatment of filovirus infections. We have previously shown that synthetic antisense phosphorodiamidate morpholino oligomers (PMOs), which target EBOV-specific transcripts, confer partial protection to infected rodents and rhesus macaques. In this report, we describe the efficacy of a new class of positively charged PMOs (PMO+).

\section{Methods}

Using established rodent models of EBOV and MARV infections, we screened virus-specific $\mathrm{PMO}+$ agents to identify combinations capable of protecting against lethal challenge. Efficacious $\mathrm{PMO}+$ combinations were further evaluated in non-human primate models of filovirus infection.

\section{Results}

In EBOV models of infection, $\mathrm{PMO}+$ molecules specific to EBOV VP35 and VP24, the combination of which is designated as AVI-6002, protected EBOV-infected mice and guinea pigs. Delivered by a combination of subcutaneous (s.c) and intraperitoneal (i.p.) routes, AVI-6002 protected 5 out of 8 (63\%) of EBOV Zaire-infected rhesus macaques when delivered using a therapeutic regimen (i.e. beginning post infection). Similar survival (60\%; 3 out of 5 macaques) was observed in a separate study when AVI-6002 was delivered intravenously (i.v.). In MARV infections models, a combination treatment containing a mixture of NP- and VP24-specific PMO+ agents, designated as AVI-6003, conferred a high degree of protection to mice and guinea pigs challenged with a lethal dose of MARV. Delivered using a therapeutic regimen, AVI-6003 completely protected MARV-infected cynomolgus macaques when delivered via s.c, i.v., or a combination of s.c. and i.p. routes.

\section{Discussion}

AVI-6002 and AVI-6003 represent highly promising therapeutic candidates for treatment of filovirus infections in humans.

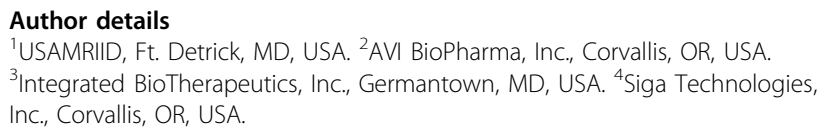

Published: 11 May 2010

doi:10.1186/1742-4690-7-S1-P4

Cite this article as: Warren et al:: Treatment of highly pathogenic filovirus infections using advanced antisense technology. Retrovirology 2010 7(Suppl 1):P4.

* Correspondence: Travis.Warren@amedd.army.mil

${ }^{1}$ USAMRIID, Ft. Detrick, MD, USA 